\title{
A novel Kind of the multi-index Beta, Gauss, and confluent hypergeometric functions
}

\author{
Musharraf Alia ${ }^{a}$, Mohd Ghayasuddin ${ }^{b}$, Waseem A. Khan ${ }^{c}$, Kottakkaran Sooppy Nisar ${ }^{\mathrm{d}, *}$ \\ ${ }^{a}$ Department of Mathematics, G.F. College, Shahjahanpur-242001, India. \\ ${ }^{b}$ Department of Mathematics, Integral University Campus, Shahjahanpur-242001, India. \\ ${ }^{c}$ Department of Mathematics and Natural Sciences, Prince Mohammad Bin Fahd University, P.O. Box: 1664, Al Khobar 31952, Saudi \\ Arabia. \\ ${ }^{d}$ Department of Mathematics, College of Arts and Sciences, Wadi Aldawser, 11991, Prince Sattam bin Abdulaziz University, Saudi \\ Arabia.
}

\begin{abstract}
This research article elaborates on a novel expansion of the beta function by using the multi-index Mittag-Leffler function. Here, we derive some basic properties of this new beta function and then present a new type of beta dispersal as an application of our proposed beta function. We also introduce a novel expansion of Gauss and confluent hypergeometric functions for our newly initiated beta function. Some important properties of our proposed hypergeometric functions (like integral representations, differential formulae, transformations formulae, summation formulae, and a generating relation) are also pointed out systematically.
\end{abstract}

Keywords: Beta function, extended beta function, Gauss hypergeometric function, confluent hypergeometric function, multi-index Mittag-Leffler function.

2020 MSC: 33B15, 33B20, 33C05, 33C15, 33E12.

(C)2021 All rights reserved.

\section{Introduction}

Innumerable primitive functions in applied sciences are explicit via improper integrals. These innumerable functions are primarily called special functions. Such functions play a discernible role in a variety of appropriate fields of science and engineering. Therefore, many researchers have demonstrated (from time to time) various extensions and analogous properties of such type of functions.

Throughout in this paper, let $\mathbb{N}, \mathbb{R}$ and $\mathbb{C}$ be the sets of natural numbers, real numbers and complex numbers, respectively, and let

$$
\mathbb{N}_{0}:=\{0,1,2,3, \ldots\}=\mathbb{N} \cup\{0\}
$$

\footnotetext{
*Corresponding author

Email addresses: drmusharrafali@gmail.com (Musharraf Ali), ghayas.maths@gmail.com (Mohd Ghayasuddin), wkhan1@pmu.edu.sa (Waseem A. Khan), n. sooppy@psau.edu.sa (Kottakkaran Sooppy Nisar)
}

doi: $10.22436 /$ jmcs.023.02.07

Received: 2020-08-01 Revised: 2020-09-08 Accepted: 2020-09-18 
The Leonhard Euler's classical beta function $\mathrm{B}\left(\zeta_{1}, \zeta_{2}\right)$ is a function of two complex variables $\zeta_{1}$ and $\zeta_{2}$ and is defined by the following integral representation (see [18], see also [20]):

$$
\mathrm{B}\left(\zeta_{1}, \zeta_{2}\right)=\int_{0}^{1} w^{\zeta_{1}-1}(1-w)^{\zeta_{2}-1} \mathrm{~d} w, \quad\left(\mathfrak{R}\left(\zeta_{1}\right)>0, \mathfrak{R}\left(\zeta_{2}\right)>0\right) .
$$

In 1997, Chaudhry et al. [3] defined the following extension of (1.1) by inserting the regularization factor $\exp \left[-\frac{\tau}{w(1-w)}\right]$ in the integrands as follows:

$$
\left.\mathrm{B}_{\tau}\left(\zeta_{1}, \zeta_{2}\right)=\int_{0}^{1} w^{\zeta_{1}-1}(1-w)^{\zeta_{2}-1} \exp \left[-\frac{\tau}{\mathcal{w}(1-w)}\right] \mathrm{d} w, \mathfrak{R}\left(\zeta_{1}\right)>0, \mathfrak{R}\left(\zeta_{2}\right)>0, \mathfrak{R}(\tau)>0\right) .
$$

Clearly, for $\tau=0,(1.2)$ reduces to (1.1). The most interesting applications of (1.2) are given by Chaudhry et al. [4]. They generalized the classical Gauss and confluent hypergeometric functions by using the extended beta function $\mathrm{B}_{\tau}\left(\zeta_{1}, \zeta_{2}\right)$ as follows:

$$
F_{\tau}\left(\kappa_{1}, \kappa_{2} ; \kappa_{3} ; u\right)=\sum_{l=0}^{\infty} \frac{\left(\kappa_{1}\right) l B_{\tau}\left(\kappa_{2}+l, \kappa_{3}-\kappa_{2}\right)}{B\left(\kappa_{2}, \kappa_{3}-\kappa_{2}\right)} \frac{u^{l}}{l !}, \quad\left(\tau \geqslant 0,|u|<1, \mathfrak{R}\left(\kappa_{3}\right)>\mathfrak{R}\left(\kappa_{2}\right)>0\right),
$$

and

$$
\Phi_{\tau}\left(\kappa_{2} ; \kappa_{3} ; u\right)=\sum_{l=0}^{\infty} \frac{B_{p}\left(\kappa_{2}+l, \kappa_{3}-\kappa_{2}\right)}{B\left(\kappa_{2}, \kappa_{3}-\kappa_{2}\right)} \frac{u^{l}}{l !}, \quad\left(\tau \geqslant 0, \Re\left(\kappa_{3}\right)>\Re\left(\kappa_{2}\right)>0\right) .
$$

In [4], the authors also defined the subsequent Euler's type integral representations of $F_{\tau}\left(\kappa_{1}, \kappa_{2} ; \kappa_{3} ; u\right)$ and $\Phi_{\tau}\left(\kappa_{2} ; \kappa_{3} ; u\right)$, respectively:

$$
\begin{gathered}
\mathrm{F}_{\tau}\left(\kappa_{1}, \kappa_{2} ; \kappa_{3} ; \mathrm{u}\right)=\frac{1}{\mathrm{~B}\left(\kappa_{2}, \kappa_{3}-\kappa_{2}\right)} \int_{0}^{1} w^{\kappa_{2}-1}(1-w)^{\kappa_{3}-\kappa_{2}-1}(1-u w)^{-\kappa_{1}} \exp \left[-\frac{\tau}{w(1-w)}\right] \mathrm{d} w, \\
\left(\tau \geqslant 0,|\arg (1-u)|<\pi, \mathfrak{R}\left(\kappa_{3}\right)>\mathfrak{R}\left(\kappa_{2}\right)>0\right),
\end{gathered}
$$

and

$$
\begin{gathered}
\Phi_{\tau}\left(\kappa_{2} ; \kappa_{3} ; u\right)=\frac{1}{\mathrm{~B}\left(\kappa_{2}, \kappa_{3}-\kappa_{2}\right)} \int_{0}^{1} w^{\kappa_{2}-1}(1-w)^{\kappa_{3}-\kappa_{2}-1} \exp \left[u w-\frac{\tau}{w(1-w)}\right] d w, \\
\left(\tau \geqslant 0, \mathfrak{R}\left(\kappa_{3}\right)>\mathfrak{R}\left(\kappa_{2}\right)>0\right) .
\end{gathered}
$$

Due to assorted applications of beta function in a wide range of engineering and sciences, a number of researchers have introduced and investigated a list of extended beta functions (see, for details, [1-3, 5$7,11,12,16,17,19])$.

In particular, Shadab et al. [19] defined the following generalization of beta function:

$$
\mathrm{B}_{\lambda}^{\tau}\left(\zeta_{1}, \zeta_{2}\right)=\int_{0}^{1} w^{\zeta_{1}-1}(1-w)^{\zeta_{2}-1} \mathrm{E}_{\lambda}\left[-\frac{\tau}{w(1-w)}\right] \mathrm{d} w, \quad\left(\lambda \in \mathbb{R}_{0}^{+}, \mathfrak{R}(\tau)>0\right),
$$

where $E_{\lambda}($.$) is the classical Mittag-Leffler function of one parameter (which is a natural extension of the$ exponential function) defined by Gosta Mittag-Leffler as follows (see [10]):

$$
\mathrm{E}_{\lambda}(w)=\sum_{k=0}^{\infty} \frac{w^{k}}{\Gamma(1+\lambda k)}, \quad(w \in \mathbb{C} \text { and } \lambda \geqslant 0) .
$$

If we put $\lambda=1$ in (1.3) then we get the extended beta function defined by (1.2). Moreover, the authors also investigated some interesting properties of $B_{\lambda}^{\tau}\left(\zeta_{1}, \zeta_{2}\right)$ in [19]. 
More recently, Ghayasuddin et al. [7] introduced the following multi-index generalization of the beta function by making use of the multi-index Mittag-Leffler function:

$$
\begin{aligned}
\mathrm{B}_{\tau}^{\left(\mathrm{a}_{1}, \ldots, \mathrm{a}_{s}, \mathrm{~b}_{1}, \ldots, \mathrm{b}_{s}\right)}\left(\zeta_{1}, \zeta_{2}\right) & =\int_{0}^{1} w^{\zeta_{1}-1}(1-w)^{\zeta_{2}-1} \mathrm{E}_{\left(\mathrm{a}_{\mathrm{i}}\right),\left(\mathrm{b}_{i}\right)}\left[-\frac{\tau}{w(1-w)}\right] \mathrm{d} w, \\
\left(\mathfrak{R}\left(\zeta_{1}\right)\right. & \left.>0, \mathfrak{R}\left(\zeta_{2}\right)>0, \mathrm{a}_{\mathrm{i}}>0, \mathrm{~b}_{\mathrm{i}} \in \mathbb{R}, \tau \geqslant 0\right)
\end{aligned}
$$

where $E_{\left(a_{i}\right),\left(b_{i}\right)}(w)$ is the multi-index (2m-parameter) Mittag-Leffler function given as follows (see $[8,9$, $21]$, see also [15]):

$$
E_{\left(a_{i}\right),\left(b_{i}\right)}(w)=\sum_{k=0}^{\infty} \frac{w^{k}}{\Gamma\left(b_{1}+k a_{1}\right) \cdots \Gamma\left(b_{s}+k a_{s}\right)},
$$

here $s>1$ is an integer and $a_{1}, \ldots, a_{s}>0$ and $b_{1}, \ldots, b_{s}$ are arbitrary real numbers. Clearly, for $s=2$, if we set $a_{1}=\lambda, a_{2}=0$, and $b_{1}=b_{2}=1$ in (1.4) then we get the extended beta function $B_{\lambda}^{\tau}\left(\zeta_{1}, \zeta_{2}\right)$ given in (1.3).

A further generalization of (1.5) so-called the multi-index (3m-parameter) Mittag-Leffler function $E_{\left(a_{i}\right),\left(b_{i}\right)}^{\left(c_{i}\right)}(w)$ is defined by (see $[13,14]$, see also [15])

$$
E_{\left(a_{i}\right),\left(b_{i}\right)}^{\left(c_{i}\right)}(w)=\sum_{k=0}^{\infty} \frac{\left(c_{1}\right)_{k} \cdots\left(c_{s}\right)_{k}}{\Gamma\left(b_{1}+k a_{1}\right) \cdots \Gamma\left(b_{s}+k a_{s}\right)} \frac{w^{k}}{(k !)^{s}},
$$

where $s>1$ be an integer, $a_{i}, b_{i}, c_{i} \in \mathbb{C}, \mathfrak{R}\left(a_{i}\right)>0$ (for every $\left.i=1,2 \ldots, s\right)$. If we set $c_{1}=c_{2}=\cdots, c_{s}=1$ in (1.6) then this multi-index (3m-parameter) Mittag-Leffler function easily reduces to the multi-index (2m-parameter) Mittag-Leffler function given in (1.5).

The main motive of the paper is to introduce a further extension of beta function $B_{\tau}^{\left(a_{1}, \cdots, a_{s}, b_{1}, \cdots, b_{s}\right)}\left(\zeta_{1}, \zeta_{2}\right)$ by making use of the multi-index (3m-parameter) Mittag-Leffler function $E_{\left(a_{i}\right),\left(b_{i}\right)}^{\left(c_{i}\right)}(w)$ and also to present a new multi-index generalization of Gauss and confluent hypergeometric functions.

\section{A new type of multi-index beta function}

In this section, we elaborate the following new multi-index beta function by making use of the multiindex Mittag-Leffler function $\mathrm{E}_{\left(\mathrm{a}_{i}\right),\left(\mathrm{b}_{\mathfrak{i}}\right)}^{\left(\mathrm{c}_{1}\right)}(w)$ :

$$
\begin{aligned}
\mathrm{B}_{\left(c_{1}, \cdots, c_{s}\right), \tau}^{\left(a_{1}, \cdots, a_{s}, b_{1}, \cdots, b_{s}\right)}\left(\zeta_{1}, \zeta_{2}\right) & =\int_{0}^{1} w^{\zeta_{1}-1}(1-w)^{\zeta_{2}-1} E_{\left(a_{i}\right),\left(b_{i}\right)}^{\left(c_{i}\right)}\left[-\frac{\tau}{w(1-w)}\right] d w, \\
\left(\Re\left(\zeta_{1}\right)\right. & \left.>0, \Re\left(\zeta_{2}\right)>0, a_{i}>0, b_{i}, c_{i} \in \mathbb{R}, \tau \geqslant 0\right) .
\end{aligned}
$$

Remark 2.1. If we set $c_{1}=c_{2}=\cdots=c_{s}=1$ in (2.1), then we get the extended beta function defined by Ghayasuddin et al. [7], which again for $s=2$, by setting $a_{1}=\lambda, a_{2}=0$, and $b_{1}=b_{2}=1$, yields the extended beta function introduced by Shadab et al. [19].

3. Integral representation of $B_{\left(c_{1}, \cdots, c_{s}\right), \tau}^{\left(a_{1}, \cdots, b_{s}, b_{1}, \cdots, b_{s}\right)}\left(\zeta_{1}, \zeta_{2}\right)$

Theorem 3.1. For $a_{i}>0, b_{i}, c_{i} \in \mathbb{R}$ and $\tau \geqslant 0$, we have the subsequent integral representations of $\mathrm{B}_{\left(\mathrm{c}_{1}, \cdots, \mathrm{c}_{s}\right), \tau}^{\left(\mathrm{a}_{1}, \cdots, \mathrm{a}_{\mathrm{s}}, \mathrm{b}_{1}, \cdots, \mathrm{b}_{s}\right)}\left(\zeta_{1}, \zeta_{2}\right)$ :

$$
\begin{aligned}
& B_{\left(c_{1}, \ldots, c_{s}\right), \tau}^{\left(a_{1}, \ldots, a_{s}, b_{1}, \ldots, b_{s}\right)}\left(\zeta_{1}, \zeta_{2}\right)=2 \int_{0}^{\frac{\pi}{2}} \cos ^{2 \zeta_{1}-1} u \sin ^{2 \zeta_{2}-1} u E_{\left(a_{i}\right),\left(b_{i}\right)}^{\left(c_{i}\right)}\left(-\tau \sec ^{2} u \csc ^{2} u\right) d u, \\
& B_{\left(c_{1}, \cdots, c_{s}\right), \tau}^{\left(a_{1}, \cdots, a_{s}, b_{1}, \cdots, b_{s}\right)}\left(\zeta_{1}, \zeta_{2}\right)=\int_{0}^{\infty} \frac{u^{\zeta_{1}-1}}{(1+u)^{\zeta_{1}+\zeta_{2}}} E_{\left(a_{i}\right),\left(b_{i}\right)}^{\left(c_{i}\right)}\left[-\tau\left(2+u+\frac{1}{u}\right)\right] d u,
\end{aligned}
$$


and

$$
\mathrm{B}_{\left(\mathrm{c}_{1}, \cdots, \mathrm{c}_{\mathrm{s}}\right), \tau}^{\left(\mathrm{a}_{1}, \cdots, \mathrm{b}_{\mathrm{s}}\right)}\left(\zeta_{1}, \zeta_{2}\right)=2^{1-\zeta_{1}-\zeta_{2}} \int_{-1}^{1}(1+u)^{\zeta_{1}-1}(1-u)^{\zeta_{2}-1} \mathrm{E}_{\left(\mathrm{a}_{\mathfrak{i}}\right),\left(\mathrm{b}_{\mathfrak{i}}\right)}^{\left(\mathrm{c}_{\mathfrak{i}}\right)}\left[-\frac{4 \tau}{\left(1-\mathrm{u}^{2}\right)}\right] \mathrm{du}
$$

Proof. On setting $w=\cos ^{2} u, w=\frac{u}{1+u}$ and $w=\frac{1+\mathfrak{u}}{2}$ in (2.1) yields, respectively, the integral representations (3.1)-(3.3).

\section{Properties of the multi-index beta function}

This section deals with some basic properties of our newly initiated multi-index beta function $\mathrm{B}_{\left(\mathrm{c}_{1}, \cdots, \mathrm{c}_{s}\right), \tau}^{\left(\mathrm{a}_{1}, \cdots, \mathrm{a}_{\mathrm{s}}, \mathrm{b}_{1}, \cdots, \mathrm{b}_{s}\right)}\left(\zeta_{1}, \zeta_{2}\right)$.

Theorem 4.1. The following identity holds true for our multi-index beta function $\mathrm{B}_{\left(\mathrm{c}_{1}, \ldots, \mathrm{c}_{s}\right), \tau}^{\left(\mathrm{a}_{1}, \ldots, \mathrm{a}_{\mathrm{s}}, \mathrm{b}_{1}, \ldots, \mathrm{b}_{s}\right)}\left(\zeta_{1}, \zeta_{2}\right)$ :

$$
\mathrm{B}_{\left(\mathrm{c}_{1}, \ldots, \mathrm{c}_{s}\right), \tau}^{\left(\mathrm{a}_{1}, \ldots, \mathrm{a}_{\mathrm{s}}, \mathrm{b}_{1}, \ldots, \mathrm{b}_{s}\right)}\left(\zeta_{1}, \zeta_{2}\right)=\sum_{k=0}^{\mathrm{l}}\left(\begin{array}{c}
\mathrm{l} \\
\mathrm{k}
\end{array}\right) \mathrm{B}_{\left(\mathrm{c}_{1}, \ldots, \mathrm{c}_{s}\right), \tau}^{\left(\mathrm{a}_{1}, \ldots, \mathrm{a}_{s}, \mathrm{~b}_{1}, \ldots, \mathrm{b}_{s}\right)}\left(\zeta_{1}+\mathrm{k}, \zeta_{2}+\mathrm{l}-\mathrm{k}\right),
$$

where $l \in \mathbb{N}_{0}$.

Proof. From (2.1), we have

$$
\begin{aligned}
& \mathrm{B}_{\left(\mathrm{c}_{1}, \ldots, \mathrm{c}_{s}\right), \tau}^{\left(\mathrm{a}_{1}, \ldots, \mathrm{a}_{\mathrm{s}}, \mathrm{b}_{1}, \ldots, \mathrm{b}_{s}\right)}\left(\zeta_{1}, \zeta_{2}\right)=\int_{0}^{1} w^{\zeta_{1}-1}(1-w)^{\zeta_{2}-1}\{w+(1-w)\} \mathrm{E}_{\left(\mathrm{a}_{\mathrm{i}}\right),\left(\mathrm{b}_{i}\right)}^{(\mathrm{c})}\left[-\frac{\tau}{w(1-w)}\right] \mathrm{d} w, \\
& \mathrm{~B}_{\left(\mathrm{c}_{1}, \ldots, \mathrm{c}_{s}\right), \tau}^{\left(\mathrm{a}_{1}, \ldots, \mathrm{a}_{s}, \mathrm{~b}_{1}, \ldots, \mathrm{b}_{s}\right)}\left(\zeta_{1}, \zeta_{2}\right)=\mathrm{B}_{\left(\mathrm{c}_{1}, \ldots, \mathrm{c}_{s}\right), \tau}^{\left(\mathrm{a}_{1}, \ldots, \mathrm{a}_{s}, \mathrm{~b}_{1}, \ldots, \mathrm{b}_{s}\right)}\left(\zeta_{1}+1, \zeta_{2}\right)+\mathrm{B}_{\left(\mathrm{c}_{1}, \ldots, \mathrm{c}_{s}\right), \tau}^{\left(\mathrm{a}_{1}, \ldots, \mathrm{b}_{1}, \ldots, \mathrm{b}_{s}\right)}\left(\zeta_{1}, \zeta_{2}+1\right) .
\end{aligned}
$$

Again, applying the same argument to each of two terms in the right side of (4.1), we get

$$
\begin{aligned}
B_{\left(c_{1}, \ldots, c_{s}\right), \tau}^{\left(a_{1}, \ldots, a_{s}, b_{1}, \ldots, b_{s}\right)}\left(\zeta_{1}, \zeta_{2}\right)= & B_{\left(c_{1}, \ldots, c_{s}\right), \tau}^{\left(a_{1}, \ldots, a_{s}, b_{1}, \ldots, b_{s}\right)}\left(\zeta_{1}+2, \zeta_{2}\right) \\
& +2 B_{\left(c_{1}, \ldots, c_{s}\right), \tau}^{\left(a_{1}, \ldots, a_{s}, b_{1}, \ldots, b_{s}\right)}\left(\zeta_{1}+1, \zeta_{2}+1\right)+B_{\left(c_{1}, \ldots, c_{s}\right), \tau}^{\left(a_{1}, \ldots, a_{s}, b_{1}, \ldots, b_{s}\right)}\left(\zeta_{1}, \zeta_{2}+2\right) .
\end{aligned}
$$

Proceeding in the same manner, induction on $k$ proves the desired result.

Theorem 4.2. The following result holds true for our multi-index beta function $\mathrm{B}_{\left(\mathrm{c}_{1}, \ldots, \mathrm{c}_{s}\right), \tau}^{\left(\mathrm{a}_{1}, \ldots, \mathrm{a}_{\mathrm{s}}, \mathrm{b}_{1}, \ldots, \mathrm{b}_{\mathrm{s}}\right)}\left(\zeta_{1}, \zeta_{2}\right)$ :

$$
\mathrm{B}_{\left(\mathrm{c}_{1}, \ldots, \mathrm{c}_{s}\right), \tau}^{\left(\mathrm{a}_{1}, \ldots, \mathrm{a}_{s}, \mathrm{~b}_{1}, \ldots, \mathrm{b}_{\mathrm{s}}\right)}\left(\zeta_{1}, 1-\zeta_{2}\right)=\sum_{\mathrm{k}=0}^{\infty} \frac{\left(\zeta_{2}\right)_{\mathrm{k}}}{\mathrm{k} !} \mathrm{B}_{\left(\mathrm{c}_{1}, \ldots, \mathrm{c}_{s}\right), \tau}^{\left(\mathrm{a}_{1}, \ldots, \mathrm{a}_{\mathrm{s}}, \mathrm{b}_{1}, \ldots, \mathrm{b}_{\mathrm{s}}\right)}\left(\zeta_{1}+\mathrm{k}, 1\right) .
$$

Proof. On using (2.1) in the left side of (4.2), we get

$$
\begin{aligned}
\mathrm{B}_{\left(\mathrm{c}_{1}, \ldots, \mathrm{c}_{s}\right), \tau}^{\left(\mathrm{a}_{1}, \ldots, \mathrm{a}_{\mathrm{s}}, \mathrm{b}_{1}, \ldots, \mathrm{b}_{s}\right)}\left(\zeta_{1}, 1-\zeta_{2}\right) & =\int_{0}^{1} w^{\zeta_{1}-1}(1-w)^{-\zeta_{2}} \mathrm{E}_{\left(\mathrm{a}_{i}\right),\left(\mathrm{b}_{i}\right)}^{\left(\mathrm{c}_{i}\right)}\left[-\frac{\tau}{w(1-w)}\right] \mathrm{d} w \\
& =\int_{0}^{1} w^{\zeta_{1}-1} \sum_{k=0}^{\infty} \frac{\left(\zeta_{2}\right)_{k} w^{k}}{k !} \mathrm{E}_{\left(\mathrm{a}_{i}\right),\left(\mathrm{b}_{i}\right)}^{\left(c_{i}\right)}\left[-\frac{\tau}{w(1-w)}\right] \mathrm{d} w .
\end{aligned}
$$

Now interchanging the order of integration and summation in the above expression and then upon using (2.1), we arrive at our claimed result. 
Theorem 4.3. The following result holds true for our multi-index beta function $\mathrm{B}_{\left(\mathrm{c}_{1}, \ldots, \mathrm{c}_{s}\right), \tau}^{\left(\mathrm{a}_{1}, \ldots, \mathrm{a}_{s}, \mathrm{~b}_{1}, \ldots, \mathrm{b}_{s}\right)}\left(\zeta_{1}, \zeta_{2}\right)$ :

$$
\mathrm{B}_{\left(\mathrm{c}_{1}, \ldots, \mathrm{c}_{s}\right), \tau}^{\left(\mathrm{a}_{1}, \ldots, \mathrm{a}_{s}, \mathrm{~b}_{1}, \ldots, \mathrm{b}_{\mathrm{s}}\right)}\left(\zeta_{1}, \zeta_{2}\right)=\sum_{k=0}^{\infty} \mathrm{B}_{\left(\mathrm{c}_{1}, \ldots, \mathrm{c}_{s}\right), \tau}^{\left(\mathrm{a}_{1}, \ldots, \mathrm{a}_{\mathrm{s}}, \mathrm{b}_{1}, \ldots, \mathrm{b}_{\mathrm{s}}\right)}\left(\zeta_{1}+\mathrm{k}, \zeta_{2}+1\right) .
$$

Proof. This theorem can be established with the help of (2.1) by writing $(1-w)^{\zeta_{2}-1}=(1-w)^{\zeta_{2}} \sum_{k=0}^{\infty} w^{k}$. We omit the details.

\section{The new beta distribution}

The beta distribution is a commonly used distribution and is frequently employed to model data. In reliability and life testing experiments, many times the data are modeled by finite range distributions. In this section, we define the following new beta distribution as an application of our multi-index beta function:

$$
f(w)= \begin{cases}C w^{\rho-1}(1-w)^{\sigma-1} E_{\left(a_{i}\right),\left(b_{i}\right)}^{\left(c_{1}\right)}\left[-\frac{\tau}{w(1-w)}\right], & (0<w<1), \\ 0, & \text { otherwise, }\end{cases}
$$

where $\rho, \sigma \in \mathbb{R}, a_{i}>0, b_{i}, c_{i} \in \mathbb{R}, \tau \geqslant 0$ and $C^{-1}=B_{\left(c_{1}, \ldots, c_{s}\right), \tau}^{\left(a_{1}, \ldots, a_{s}, b_{1}, \ldots, b_{s}\right)}(\rho, \sigma)$.

Next, we have presented here some fundamental properties of our introduced beta distribution (5.1).

For $r \in \mathbb{R}$, the $r^{\text {th }}$ moment of the p.d.f $f(w)$ about the origin is given by

$$
E\left(W^{r}\right)=\frac{B_{\left(c_{1}, \ldots, c_{s}\right), \tau}^{\left(a_{1}, \ldots, a_{s}\right)}(\rho+r, \sigma)}{B_{\left(c_{1}, \ldots, c_{s}\right), \tau}^{\left(a_{1}, \ldots, b_{s}, b_{1}, \ldots, b_{s}\right)}(\rho, \sigma)}, \quad\left(\rho, \sigma \in \mathbb{R}, a_{i}>0, b_{i}, c_{i} \in \mathbb{R}, \tau \geqslant 0\right) .
$$

The specific case of (5.2) for $r=1$ yields the mean of our proposed beta distribution (5.1):

$$
E(W)=\frac{B_{\left(c_{1}, \ldots, c_{s}, \tau\right.}^{\left(a_{1}, \ldots, b_{s}\right)}(\rho+1, \sigma)}{B_{\left(c_{1}, \ldots, c_{s}\right), \tau}^{\left(a_{1}, \ldots, b_{s}, b_{1,}, \ldots, b_{s}\right)}(\rho, \sigma)} .
$$

The variance of our beta distribution is defined by

$$
\begin{aligned}
& \operatorname{Var}(W)=E\left(W^{2}\right)-[E(W)]^{2}, \\
& \operatorname{Var}(W)=\frac{B_{\left(c_{1}, \ldots, c_{s}\right), \tau}^{\left(a_{1}, \ldots, a_{s}, b_{1}\right)}(\rho+2, \sigma) B_{\left(c_{1}, \ldots, c_{s}\right), \tau}^{\left(a_{1}, \ldots, a_{s}, b_{1}, \ldots, b_{s}\right)}(\rho, \sigma)-\left[B_{\left(c_{1}, \ldots, c_{s}\right), \tau}^{\left(a_{1}, \ldots, a_{s}, b_{1}, \ldots, b_{s}\right)}(\rho+1, \sigma)\right]^{2}}{\left[B_{\left(c_{1}, \ldots, c_{s}\right), \tau}^{\left(a_{1}, \ldots, a_{1}, b_{1}, \ldots, b_{s}\right)}(\rho, \sigma)\right]^{2}} .
\end{aligned}
$$

The coefficient of variation of our introduced distribution (which is defined as the ratio of the standard deviation and mean) can be expressed as

$$
C . V=\sqrt{\frac{B_{\left(c_{1}, \ldots, c_{s}\right), \tau}^{\left(a_{1}, \ldots, a_{s}, b_{1}, \ldots, b_{s}\right)}(\rho+2, \sigma) B_{\left(c_{1}, \ldots, c_{s}\right), \tau}^{\left(a_{1}, \ldots, a_{s}, b_{1}, \ldots, b_{s}\right)}(\rho, \sigma)}{\left[B_{\left(c_{1}, \ldots, c_{s}\right), \tau}^{\left(a_{1}, \ldots, a_{s}, b_{1,}, \ldots, b_{s}\right)}(\rho+1, \sigma)\right]^{2}}}-1 .
$$

The moment generating function (m.g.f) about origin of our extended beta distribution is given by

$$
M_{W}(t)=\sum_{r=0}^{\infty} \frac{t^{r}}{r !} E\left(W^{r}\right), \quad M_{W}(t)=\frac{1}{B_{\left(c_{1}, \ldots, c_{s}\right), \tau}^{\left(a_{1}, \ldots, a_{1}, b_{1}, \ldots, b_{s}\right)}(\rho, \sigma)} \sum_{r=0}^{\infty} B_{\left(c_{1}, \ldots, c_{s}\right), \tau}^{\left(a_{1}, \ldots, a_{s}, b_{1}, \ldots, b_{s}\right)}(\rho+r, \sigma) \frac{t^{r}}{r !} .
$$


The characteristic function of our proposed distribution can be calculated as follows:

$$
E\left(e^{i t w}\right)=\sum_{r=0}^{\infty} \frac{i^{r} t^{r}}{r !} E\left(W^{r}\right), \quad E\left(e^{i t w}\right)=\frac{1}{B_{\left(c_{1}, \ldots, c_{s}\right), \tau}^{\left(a_{1}, \ldots, a_{s}, b_{1}, \ldots, b_{s}\right)}(\rho, \sigma)} \sum_{r=0}^{\infty} B_{\left(c_{1}, \ldots, c_{s}\right), \tau}^{\left(a_{1}, \ldots, a_{s}, b_{1}, \ldots, b_{s}\right)}(\rho+r, \sigma) \frac{i^{r} t^{r}}{r !} .
$$

The cumulative distribution function of our beta distribution (5.1) can be expressed as

$$
\mathrm{F}(w)=\mathrm{P}[\mathrm{W}<w]=\int_{0}^{w} \mathrm{f}(w) \mathrm{d} w, \quad \mathrm{~F}(w)=\frac{\mathrm{B}_{\left(\mathrm{c}_{1}, \ldots, \mathrm{c}_{s}\right), \tau, w}^{\left(\mathrm{a}_{1}, \ldots, \mathrm{b}_{\mathrm{s}}, \mathrm{b}_{1}, \ldots, \mathrm{b}_{\mathrm{s}}\right)}(\rho, \sigma)}{\mathrm{B}_{\left(\mathrm{c}_{1}, \ldots, \mathrm{c}_{\mathrm{s}}\right), \tau}^{\left(\mathrm{a}_{1}, \ldots, \mathrm{b}_{s}\right)}(\rho, \sigma)},
$$

where $B_{\left(c_{1}, \ldots, c_{s}\right), \tau, w}^{\left(a_{1}, \ldots, a_{s}, b_{1}, \ldots, b_{s}\right)}(\rho, \sigma)$ denotes the new incomplete beta function defined by

$$
\mathrm{B}_{\left(\mathrm{c}_{1}, \ldots, \mathrm{c}_{s}\right), \tau, w}^{\left(\mathrm{a}_{1}, \ldots, \mathrm{a}_{\mathrm{s}}, \mathrm{b}_{1}, \ldots, \mathrm{b}_{s}\right)}(\rho, \sigma)=\int_{0}^{w} w^{\rho-1}(1-w)^{\sigma-1} \mathrm{E}_{\left(\mathrm{a}_{i}\right),\left(\mathrm{b}_{i}\right)}^{\left(c_{i}\right)}\left[-\frac{\tau}{w(1-w)}\right] \mathrm{d} w .
$$

The reliability function (which is simply the complement of the cumulative distribution function) of our newly introduced distribution is given by

$$
R(w)=P[W \geqslant w]=1-F(w)=\int_{w}^{\infty} f(w) d w, \quad R(w)=\frac{B_{\left(c_{1}, \ldots, c_{s}\right), \tau, w}^{\left(a_{1}, \ldots, b_{s}\right)}(\rho, \sigma)}{B_{\left(c_{1}, \ldots, c_{s}\right), \tau}^{\left(a_{1}, \ldots, a_{s}, b_{1}, \ldots, b_{s}\right)}(\rho, \sigma)}
$$

where $B_{\left(c_{1}, \ldots, c_{s}\right), \tau, w}^{\left(a_{1}, \ldots, a_{s}, b_{1}, \ldots, b_{s}\right)}(\rho, \sigma)$ is the incomplete beta function defined by

$$
\mathrm{B}_{\left(c_{1}, \ldots, c_{s}\right), \tau, w}^{\left(a_{1}, \ldots, a_{s}, b_{1}, \ldots, b_{s}\right)}(\rho, \sigma)=\int_{w}^{\infty} w^{\rho-1}(1-w)^{\sigma-1} E_{\left(a_{i}\right),\left(b_{i}\right)}^{\left(c_{i}\right)}\left[-\frac{\tau}{w(1-w)}\right] d w .
$$

\section{Multi-index hypergeometric functions and their associated properties}

In this section, we present the following multi-index Gauss and confluent hypergeometric functions by making use of our introduced multi-index beta function $B_{\left(c_{1}, \ldots, c_{s}\right), \tau}^{\left(a_{1}, \ldots, a_{s}, b_{1}, \ldots, b_{s}\right)}\left(\zeta_{1}, \zeta_{2}\right)$ :

$$
\begin{gathered}
F_{\left(c_{1}, \ldots, c_{s}\right), \tau}^{\left(a_{1}, \ldots, a_{s}, b_{1}, \ldots, b_{s}\right)}\left(\kappa_{1}, \kappa_{2} ; \kappa_{3} ; u\right)=\sum_{l=0}^{\infty} \frac{\left(\kappa_{1}\right)_{l} B_{\left(c_{1}, \ldots, c_{s}\right), \tau}^{\left(a_{1}, \ldots, a_{s}, b_{1}, \ldots, b_{s}\right)}\left(\kappa_{2}+l, \kappa_{3}-\kappa_{2}\right)}{B\left(\kappa_{2}, \kappa_{3}-\kappa_{2}\right)} \frac{u^{l}}{l !} \\
\left(\tau \geqslant 0,|u|<1, \mathfrak{R}\left(\kappa_{3}\right)>\Re\left(\kappa_{2}\right)>0, a_{i}>0, b_{i}, c_{i} \in \mathbb{R}\right)
\end{gathered}
$$

and

$$
\begin{gathered}
\Phi_{\left(c_{1}, \ldots, c_{s}\right), \tau}^{\left(a_{1}, \ldots, a_{s}, b_{1}, \ldots, b_{s}\right)}\left(\kappa_{2} ; \kappa_{3} ; u\right)=\sum_{l=0}^{\infty} \frac{B_{\left(c_{1}, \ldots, c_{s}\right), \tau}^{\left(a_{1}, \ldots, a_{s}, b_{1}, \ldots, b_{s}\right)}\left(\kappa_{2}+l, \kappa_{3}-\kappa_{2}\right)}{B\left(\kappa_{2}, \kappa_{3}-\kappa_{2}\right)} \frac{u^{l}}{l !} \\
\left(\tau \geqslant 0, \Re\left(\kappa_{3}\right)>\Re\left(\kappa_{2}\right)>0, a_{i}>0, b_{i}, c_{i} \in \mathbb{R}\right) .
\end{gathered}
$$

Remark 6.1. On setting $c_{1}=c_{2}=\cdots=c_{s}=1,(6.1)$ and (6.2) reduce to the known extensions of Gauss and confluent hypergeometric functions defined by Ghayasuddin et al. [7], which further for $s=2$, with $a_{1}=1, a_{2}=0$, and $b_{1}=b_{2}=1$, yields the known extensions of Gauss and confluent hypergeometric functions defined by Shadab et al. [19].

Theorem 6.2. The following integral representations of our multi-index Gauss and confluent hypergeometric functions holds true:

$$
\mathrm{F}_{\left(\mathrm{c}_{1}, \ldots, \mathrm{c}_{s}\right), \tau}^{\left(\mathrm{a}_{1}, \ldots, \mathrm{c}_{\mathrm{s}}, \mathrm{b}_{1}, \ldots, \mathrm{b}_{s}\right)}\left(\mathrm{k}_{1}, \mathrm{k}_{2} ; \mathrm{k}_{3} ; \mathrm{u}\right)
$$




$$
\begin{aligned}
& =\frac{1}{\mathrm{~B}\left(\kappa_{2}, \kappa_{3}-\kappa_{2}\right)} \int_{0}^{1} w^{\kappa_{2}-1}(1-w)^{\kappa_{3}-\kappa_{2}-1}(1-u w)^{-\kappa_{1}} \mathrm{E}_{\left(\mathrm{a}_{i}\right),\left(\mathrm{b}_{i}\right)}^{\left(c_{i}\right)}\left[-\frac{\tau}{w(1-w)}\right] \mathrm{d} w, \\
& \left(\tau \geqslant 0,|\arg (1-u)|<\pi, \mathfrak{R}\left(\kappa_{3}\right)>\mathfrak{R}\left(\kappa_{2}\right)>0, a_{i}>0, b_{i}, c_{i} \in \mathbb{R}\right)
\end{aligned}
$$

and

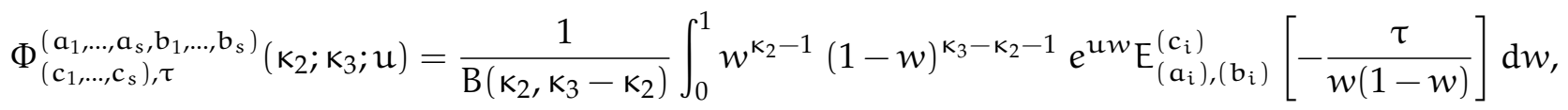

$$
\begin{aligned}
& \left(\tau \geqslant 0, \mathfrak{R}\left(\kappa_{3}\right)>\mathfrak{R}\left(\kappa_{2}\right)>0, a_{i}>0, b_{i}, c_{i} \in \mathbb{R}\right) \text {. }
\end{aligned}
$$

Proof. Each of the above integral representations can be easily established by using the integral representation of our multi-index beta function (2.1) in the right sides of (6.1) and (6.2), respectively.

Theorem 6.3. The following differential formulas for our multi-index Gauss and confluent hypergeometric functions holds true:

$$
\begin{aligned}
& \frac{d^{m}}{d u^{m}}\left\{F_{\left(c_{1}, \ldots, c_{s}\right), \tau}^{\left(a_{1}, \ldots, b_{s}, b_{1}, \ldots, b_{s}\right)}\left(\kappa_{1}, \kappa_{2} ; \kappa_{3} ; u\right)\right\}=\frac{\left(k_{1}\right)_{m}\left(\kappa_{2}\right)_{m}}{\left(\kappa_{3}\right)_{m}} F_{\left(c_{1}, \ldots, c_{s}\right), \tau}^{\left(a_{1}, \ldots, a_{s}, b_{1}, \ldots, b_{s}\right)}\left(k_{1}+m, k_{2}+m ; \kappa_{3}+m ; u\right), \\
& \left(\tau \geqslant 0, a_{i}>0, b_{i}, c_{i} \in \mathbb{R}, m \in \mathbb{N}_{0}\right)
\end{aligned}
$$

and

$$
\begin{gathered}
\frac{d^{m}}{d u^{m}}\left\{\Phi_{\left(c_{1}, \ldots, c_{s}\right), \tau}^{\left(a_{1}, \ldots, a_{s}, b_{1}, \ldots, b_{s}\right)}\left(\kappa_{2} ; \kappa_{3} ; u\right)\right\}=\frac{\left(\kappa_{2}\right)_{m}}{\left(\kappa_{3}\right)_{m}} \Phi_{\left(c_{1}, \ldots, c_{s}\right), \tau}^{\left(a_{1}, \ldots, a_{s}, b_{1}, \ldots, b_{s}\right)}\left(\kappa_{2}+m ; \kappa_{3}+m ; u\right), \\
\left(\tau \geqslant 0, a_{i}>0, b_{i}, c_{i} \in \mathbb{R}, m \in \mathbb{N}_{0}\right) .
\end{gathered}
$$

Proof. On differentiating (6.1) with respect to $u$, we get

$$
\frac{d}{d u}\left\{F_{\left(c_{1}, \ldots, c_{s}\right), \tau}^{\left(a_{1}, \ldots, a_{s}, b_{1}, \ldots, b_{s}\right)}\left(\kappa_{1}, \kappa_{2} ; \kappa_{3} ; u\right)\right\}=\sum_{l=1}^{\infty} \frac{\left(\kappa_{1}\right) l B_{\left(c_{1}, \ldots, c_{s}\right), \tau}^{\left(a_{1}, \ldots, a_{s}, b_{1}, \ldots, b_{s}\right)}\left(\kappa_{2}+l, \kappa_{3}-\kappa_{2}\right)}{B\left(\kappa_{2}, \kappa_{3}-\kappa_{2}\right)} \frac{u^{l-1}}{(l-1) !} .
$$

On replacing $l$ by $l+1$, we have

$$
\frac{d}{d u}\left\{F_{\left(c_{1}, \ldots, c_{s}\right), \tau}^{\left(a_{1}, \ldots, a_{s}, b_{1}, \ldots, b_{s}\right)}\left(\kappa_{1}, \kappa_{2} ; \kappa_{3} ; u\right)\right\}=\sum_{l=0}^{\infty} \frac{\left(\kappa_{1}\right)_{l+1} B_{\left(c_{1}, \ldots, c_{s}\right), \tau}^{\left(a_{1}, \ldots, a_{s}, b_{1}, \ldots, b_{s}\right)}\left(\kappa_{2}+l+1, \kappa_{3}-\kappa_{2}\right)}{B\left(\kappa_{2}, \kappa_{3}-\kappa_{2}\right)} \frac{u^{l}}{l !} .
$$

Now, by using $B\left(\kappa_{2}, \kappa_{3}-\kappa_{2}\right)=\frac{\kappa_{3}}{\kappa_{2}} B\left(\kappa_{2}+1, \kappa_{3}-\kappa_{2}\right)$ and $\left(\kappa_{1}\right)_{l+1}=\kappa_{1}\left(\kappa_{1}+1\right)_{l}$, we get

$$
\begin{aligned}
\frac{d}{d u}\left\{F_{\left(c_{1}, \ldots, c_{s}\right), \tau}^{\left(a_{1}, \ldots, a_{s}, b_{1}, \ldots, b_{s}\right)}\left(\kappa_{1}, \kappa_{2} ; \kappa_{3} ; u\right)\right\} & =\frac{\kappa_{1} \kappa_{2}}{\kappa_{3}} \sum_{l=0}^{\infty} \frac{\left(\kappa_{1}+1\right){ }_{l} B_{\left(c_{1}, \ldots, c_{s}\right), \tau}^{\left(a_{1}, \ldots, b_{s}, b_{1}, \ldots, b_{s}\right)}\left(\kappa_{2}+l+1, \kappa_{3}-\kappa_{2}\right)}{B\left(\kappa_{2}+1, \kappa_{3}-\kappa_{2}\right)} \frac{u^{l}}{l !} \\
& =\frac{\kappa_{1} \kappa_{2}}{\kappa_{3}} F_{\left(c_{1}, \ldots, c_{s}\right), \tau}^{\left(a_{1}, \ldots, a_{s}, b_{1}, \ldots, b_{s}\right)}\left(\kappa_{1}+1, \kappa_{2}+1 ; \kappa_{3}+1 ; u\right) .
\end{aligned}
$$

Further, on differentiating (6.6) with respect to $u$, we get

$$
\frac{d^{2}}{d u^{2}}\left\{F_{\left(c_{1}, \ldots, c_{s}\right), \tau}^{\left(a_{1}, \ldots, a_{s}, b_{1}, \ldots, b_{s}\right)}\left(\kappa_{1}, k_{2} ; k_{3} ; u\right)\right\}=\frac{\kappa_{1}\left(k_{1}+1\right) \kappa_{2}\left(\kappa_{2}+1\right)}{k_{3}\left(\kappa_{3}+1\right)} F_{\left(c_{1}, \ldots, c_{s}\right), \tau}^{\left(a_{1}, \ldots, a_{s}, b_{1}, \ldots, b_{s}\right)}\left(\kappa_{1}+2, \kappa_{2}+2 ; k_{3}+2 ; u\right) .
$$

Proceeding in the same manner, by induction, we obtain our claimed result (6.4). Similarly, we can establish the result (6.5). 
Theorem 6.4. The following transformation formulas for our multi-index Gauss and confluent hypergeometric functions holds true:

$$
\begin{aligned}
& F_{\left(c_{1}, \ldots, c_{s}\right), \tau}^{\left(a_{1}, \ldots, a_{s}, b_{1}, \ldots, b_{s}\right)}\left(\kappa_{1}, \kappa_{2} ; \kappa_{3} ; u\right)=(1-u)^{-\kappa_{1}} F_{\left(c_{1}, \ldots, c_{s}\right), \tau}^{\left(a_{1}, \ldots, a_{s}, b_{1}, \ldots, b_{s}\right)}\left(\kappa_{1}, \kappa_{3}-\kappa_{2} ; \kappa_{2} ;-\frac{u}{(1-u)}\right), \\
& \left(\tau \geqslant 0, a_{i}>0, b_{i}, c_{i} \in \mathbb{R}\right)
\end{aligned}
$$

and

$$
\Phi_{\left(c_{1}, \ldots, c_{s}\right), \tau}^{\left(a_{1}, \ldots, a_{s}, b_{1}, \ldots, b_{s}\right)}\left(\kappa_{2} ; k_{3} ; u\right)=e^{u} \Phi_{\left(c_{1}, \ldots, c_{s}\right), \tau}^{\left(a_{1}, \ldots, a_{s}, b_{1}, \ldots, b_{s}\right)}\left(\kappa_{3}-\kappa_{2} ; \kappa_{3} ;-u\right), \quad\left(\tau \geqslant 0, a_{i}>0, b_{i}, c_{i} \in \mathbb{R}\right) .
$$

Proof. On replacing $w$ by $1-w$ in (6.3) and then by using $[1-\mathfrak{u}(1-w)]^{-\kappa_{1}}=(1-\mathfrak{u})^{-\kappa_{1}}\left[1+\frac{u}{1-u} w\right]^{-\kappa_{1}}$, we obtain

$$
\begin{aligned}
& \mathrm{F}_{\left(\mathrm{c}_{1}, \ldots, \mathrm{c}_{s}\right), \tau}^{\left(\mathrm{a}_{1}, \ldots, \mathrm{a}_{\mathrm{s}}, \mathrm{b}_{1}, \ldots, \mathrm{b}_{\mathrm{s}}\right)}\left(\mathrm{k}_{1}, \mathrm{k}_{2} ; \mathrm{k}_{3} ; \mathrm{u}\right)
\end{aligned}
$$

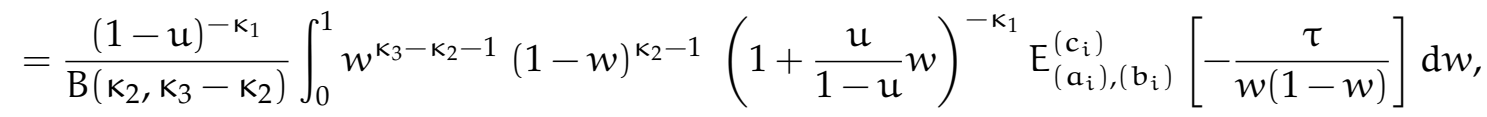

which further on using (6.3), yields our needed result (6.7). In a similar way, we can establish (6.8).

Theorem 6.5. The following summation formula for our multi-index Gauss hypergeometric function holds true:

$$
\begin{array}{r}
F_{\left(c_{1}, \ldots, c_{s}\right), \tau}^{\left(a_{1}, \ldots, a_{s}, b_{1}, \ldots, b_{s}\right)}\left(\kappa_{1}, \kappa_{2} ; \kappa_{3} ; 1\right)=\frac{B_{\left(c_{1}, \ldots, c_{s}\right), \tau}^{\left(a_{1}, \ldots, a_{s}, b_{1}, \ldots, b_{s}\right)}\left(\kappa_{2}, \kappa_{3}-\kappa_{1}-\kappa_{2}\right)}{B\left(\kappa_{2}, \kappa_{3}-\kappa_{2}\right)}, \\
\left(\tau \geqslant 0, a_{i}>0, b_{i}, c_{i} \in \mathbb{R}, \mathfrak{R}\left(\kappa_{3}-\kappa_{1}-\kappa_{2}\right)>0\right) .
\end{array}
$$

Proof. On putting $u=1$ in (6.3) and then by using (2.1), we get our required result (6.9).

Theorem 6.6. The following generating function for our multi-index Gauss hypergeometric function holds true:

$$
\begin{gathered}
\sum_{l=0}^{\infty}(c)_{l} F_{\left(c_{1}, \ldots, c_{s}\right), \tau}^{\left(a_{1}, \ldots, a_{s}, b_{1}, \ldots, b_{s}\right)}\left(\kappa_{1}+l, \kappa_{2} ; \kappa_{3} ; u\right) \frac{w^{l}}{l !}=(1-w)^{-\kappa_{1}} F_{\left(c_{1}, \ldots, c_{s}\right), \tau}^{\left(a_{1}, \ldots, a_{s}, b_{1}, \ldots, b_{s}\right)}\left(\kappa_{1}, \kappa_{2} ; \kappa_{3} ; \frac{u}{1-w}\right), \\
\left(\tau \geqslant 0, a_{i}>0, b_{i}, c_{i} \in \mathbb{R},|w|<1\right) .
\end{gathered}
$$

Proof. Using (6.1) on the left side of (6.10), we have

$$
\begin{aligned}
& \sum_{l=0}^{\infty}(c)_{l} F_{\left(c_{1}, \ldots, c_{s}\right), \tau}^{\left(a_{1}, \ldots, a_{s}, b_{1}, \ldots, b_{s}\right)}\left(\kappa_{1}+l, \kappa_{2} ; \kappa_{3} ; u\right) \frac{w^{l}}{l !} \\
& \quad=\sum_{l=0}^{\infty}(c)_{l}\left[\sum_{n=0}^{\infty} \frac{\left(\kappa_{1}+l\right)_{n} B_{\left(c_{1}, \ldots, c_{s}\right), \tau}^{\left(a_{1}, \ldots, a_{2}, b_{1}, \ldots, b_{s}\right)}\left(\kappa_{2}+n, \kappa_{3}-\kappa_{2}\right)}{B\left(\kappa_{2}, \kappa_{3}-\kappa_{2}\right)} \frac{u^{n}}{n !}\right] \frac{w^{l}}{l !} .
\end{aligned}
$$

Now, by using the identity $(c)_{n}(c+n)_{l}=(c)_{l}(c+l)_{n}$, in the above expression, we obtain

$$
\begin{aligned}
\sum_{l=0}^{\infty}(c)_{l} F_{\left(c_{1}, \ldots, c_{s}\right), \tau}^{\left(a_{1}, \ldots, a_{s}, b_{1}, \ldots, b_{s}\right)}\left(\kappa_{1}+l, \kappa_{2} ; \kappa_{3} ; u\right) \frac{w^{l}}{l !} & \\
= & \sum_{n=0}^{\infty}(c)_{n} \frac{B_{\left(c_{1}, \ldots, c_{s}\right), \tau}^{\left(a_{1}, \ldots, b_{s}, b_{s}\right)}\left(\kappa_{2}+n, \kappa_{3}-\kappa_{2}\right)}{B\left(\kappa_{2}, \kappa_{3}-\kappa_{2}\right)}\left[\sum_{l=0}^{\infty}\left(\kappa_{1}+n\right) l \frac{w^{l}}{l !}\right] \frac{u^{n}}{n !} \\
= & \sum_{n=0}^{\infty}(c)_{n} \frac{B_{\left(c_{1}, \ldots, c_{s}\right), \tau}^{\left(a_{1}, \ldots, a_{s}, b_{1}, \ldots, b_{s}\right)}\left(\kappa_{2}+n, \kappa_{3}-\kappa_{2}\right)}{B\left(\kappa_{2}, \kappa_{3}-\kappa_{2}\right)}(1-w)^{-\left(\kappa_{1}+n\right)} \frac{u^{n}}{n !}
\end{aligned}
$$

which, in view of (6.1), yields our claimed result (6.10). 


\section{Concluding remarks}

In this article, we have elaborated a novel expansion of beta function by means of the multi-index Mittag-Leffler function. First we have derived some basic properties of our introduced beta function and then we have presented a new type of beta distribution as an application of our proposed beta function. We have also defined a novel expansion of Gauss and confluent hypergeometric functions with respect to our new multi-index beta function. Next, we have indicated some analytical properties of our introduced hypergeometric functions in a systematic manner.

Furthermore, in this section, we briefly discuss about the variations in the definition of our proposed multi-index beta function $B_{\left(c_{1}, \ldots, c_{s}\right), \tau}^{\left(a_{1}, \ldots, a_{s}, b_{1}, \ldots, b_{s}\right)}\left(\zeta_{1}, \zeta_{2}\right)$.

The multi-index Mittag-Leffler function $\mathrm{E}_{\left(a_{i}\right),\left(b_{i}\right)}^{\left(c_{i}\right)}(w)$ have the following relations with Wright hypergeometric function $\Psi_{q}(w)$ and Fox H-function $\mathrm{H}_{p, q}^{m, n}(w)$ (see for details [14]):

$$
E_{\left(a_{i}\right),\left(b_{i}\right)}^{\left(c_{i}\right)}(w)=\left[\prod_{i=1}^{m} \Gamma\left(\gamma_{i}\right)\right]^{-1}{ }_{s} \Psi_{2 s-1}\left[\begin{array}{cc}
\left(c_{i}, 1\right)_{1}^{s} & \mid w \\
\left(b_{i}, a_{i}\right)_{1}^{s},(1,1)_{1}^{s-1}
\end{array}\right]
$$

and

$$
E_{\left(a_{i}\right),\left(b_{i}\right)}^{\left(c_{i}\right)}(w)=\left[\prod_{i=1}^{m} \Gamma\left(\gamma_{i}\right)\right]^{-1} H_{m, 2 m}^{1, m}\left[\begin{array}{cc}
\left(1-\gamma_{1}, 1\right) \cdots\left(1-\gamma_{m}, 1\right) \\
-w \mid & {\left[(0,1),\left(1-b_{i}, a_{i}\right)\right]_{1}^{s}}
\end{array}\right] .
$$

Therefore, our new multi-index beta function given in (2.1), is easily converted in terms of Wright hypergeometric function and Fox H-function as follows:

$$
\begin{aligned}
\mathrm{B}_{\left(c_{1}, \ldots, c_{s}\right), \tau}^{\left(a_{1}, \ldots, a_{s}, b_{1}, \ldots, b_{s}\right)}\left(\zeta_{1}, \zeta_{2}\right)= & \int_{0}^{1} w^{\zeta_{1}-1}(1-w)^{\zeta_{2}-1}\left[\prod_{i=1}^{m} \Gamma\left(\gamma_{i}\right)\right]^{-1} \\
& \times{ }_{s} \Psi_{2 s-1}\left[\begin{array}{c}
\left(c_{i}, 1\right)_{1}^{s} \\
\left(b_{i}, a_{i}\right)_{1}^{s},(1,1)_{1}^{s-1}
\end{array} \frac{\tau}{w(1-w)}\right] d w
\end{aligned}
$$

and

$$
\begin{aligned}
\mathrm{B}_{\left(\mathrm{c}_{1}, \ldots, \mathrm{c}_{s}\right), \tau}^{\left(\mathrm{a}_{1}, \ldots, \mathrm{a}_{\mathrm{s}}, \mathrm{b}_{1}, \ldots, \mathrm{b}_{s}\right)}\left(\zeta_{1}, \zeta_{2}\right)= & \int_{0}^{1} w^{\zeta_{1}-1}(1-w)^{\zeta_{2}-1}\left[\prod_{i=1}^{m} \Gamma\left(\gamma_{i}\right)\right]^{-1} \\
& \times \mathrm{H}_{m, 2 m}^{1, m}\left[\begin{array}{cc}
-\left.\frac{\tau}{w(1-w)}\right|^{\left(1-\gamma_{1}, 1\right) \cdot \cdot\left(1-\gamma_{m}, 1\right)} & {\left[(0,1),\left(1-b_{i}, a_{i}\right)\right]_{1}^{s}}
\end{array}\right] \mathrm{d} w .
\end{aligned}
$$

Note that, by using the relations (7.1) and (7.2), we can also define some variations in the integral representations of our introduced multi-index Gauss and confluent hypergeometric functions.

\section{Acknowledgment}

The author K. S. Nisar thanks to the Deanship of Scientific Research (DSR), Prince Sattam bin Abdulaziz University, Saudi Arabia for providing facilities and support.

\section{References}

[1] A. A. Al-Gonah, W. K. Mohammed, A new extension of extended gamma and beta functions and their properties, J. Sci. Eng. Res., 9 (2018), 257-270. 1 
[2] M. Ali, M. Ghayasuddin, A note on extended beta, Gauss and confluent hypergeometric functions, Italian J. pure Appl. Math., (Accepted).

[3] M. A. Chaudhry, A. Qadir, M. Rafique, S. M. Zubair, Extension of Euler's beta function, J. Comput. Appl. Math., 78 (1997), 19-32. 1, 1

[4] M. A. Chaudhry, A. Qadir, H. M. Srivastava, R. B. Paris, Extended hypergeometric and confluent hypergeometric functions, Appl. Math. Comput., 159 (2004), 589-602. 1, 1

[5] J. Choi, A. K. Rathie, R. K. Parmar, Extension of extended beta, hypergeometric and confluent hypergeometric functions, Honam Math. J., 36 (2014), 357-385. 1

[6] M. Ghayasuddin, N. U. Khan, M. Ali, A unified presentation of beta, Gauss and confluent hypergeometric functions, (communicated), (2019).

[7] M. Ghayasuddin, N. U. Khan, M. Ali, A study of extended beta, Gauss and confluent hypergeometric function, Inter. J. Appl. Math., 33 (2020), 1-13. 1, 1, 2.1, 6.1

[8] V. S. Kiryakova, Multiple (multiindex) Mittag-Leffler functions and relations to generalized fractional calculus, J. Comput. Appl. Math., 118 (2000), 241-259. 1

[9] V. S. Kiryakova, The multi-index Mittag-Leffler functions as an important class of special functions of fractional calculus, Comput. Math. Appl., 59 (2010), 1885-1895. 1

[10] G. M. Mittag-Leffler, Sur la représentation analytique d'une branche uniforme d'une fonction monogéne, Acta Math., 29 (1905), 101-182. 1

[11] S. Mubeen, G. Rahman, K. S. Nisar, J. Choi, M. Arshad, An extended beta function and its properties, Far East J. Math. Sci., 102 (2017), 1545-1557. 1

[12] E. Özergin, M. A. Özarslan, A. Altın, Extension of gamma, beta and hypergeometric functions, J. Comput. Appl. Math., 235 (2011), 4601-4610. 1

[13] J. Paneva-Konovska, Multi-index (3m-parametric) Mittag-Leffler functions and fractional calculus, C. R. Acad. Bulgare Sci., 64 (2011), 1089-1098. 1

[14] J. Paneva-Konovska, From Bessel to multi-index Mittag-Leffler functions: Enumerable families, series in them and convergence, World Scientific Publishing, London, (2016). 1, 7

[15] J. Paneva-Konovska, A survey on Bessel type functions as multi-index Mittag-Leffler functions: Differential and integral relations, Int. J. Appl. Math., 32 (2019), 357-380. 1, 1

[16] R. K. Parmar, A new generalization of gamma, beta, hypergeometric and confluent hypergeometric functions, Matematiche (Catania), 68 (2013), 33-52. 1

[17] P. I. Pucheta, A new extended beta function, Int. J. Math. Appl., 5 (2017), 255-260. 1

[18] E. D. Rainville, Special functions, Macmillan Co., New York, (1971). 1

[19] M. Shadab, S. Jabee, J. Choi, An extended beta function and its application, Far East J. Math. Sci., 103 (2018), $235-251$. $1,1,2.1,6.1$

[20] H. M. Srivastava, H. L. Manocha, A treatise on generating functions, John Wiley \& Sons, New York, (1984). 1

[21] S. B. Yakubovich, Y. F. Luchko, The hypergeometric approach to integral transform and convolutions, Kluwer Academic Publishers Group, Dordrecht, (1994). 1 